\title{
Analysis of automobile water tank support structure based on computer software
}

\author{
Jinsong $\mathrm{Li}^{1, \mathrm{a}}$, Mei $\mathrm{Li}^{1, \mathrm{~b}}$, Ye Mei ${ }^{1, \mathrm{c}}$ and Zhiyang $\mathrm{Jin}^{1, \mathrm{~d}}$ \\ ${ }^{1}$ College of Mechanical and Electrical Engineering, Hainan University, Haikou, 570228, China

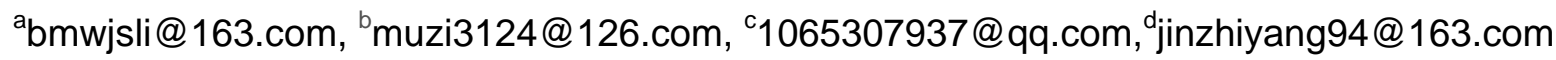

Keywords: computer, auto radiator support, FEA, Catia, UG, Ansys.

\begin{abstract}
A radiator bracket is installed on the bracket of an automobile water tank, water tank with support, and cushioning effect. This paper illustrates a Haikou car company of polypropylene and glass fiber tank material support as an example, and the use of Catia, UG and ANSYS software to analyze the structural strength, stiffness and dynamic characteristics, so as to determine whether to meet the requirements of use. First of all, the water tank model is established by the actual use of Catia software; then, the model into UG software, ANSYS software for the finite element static analysis; finally, according to the related computer software to analysis the results concluded. The results show that, polypropylene and glass fiber tank material support meets the using requirements and the water tank bracket compares to traditional materials has more advantages.
\end{abstract}

\section{Introduction}

The advent of the automobile has changed the way people travel, affecting people's life involved in related fields. After the cast iron to the alloy to the composite material, the car has gradually reduced weight. The new composite material, the emergence of polypropylene glass fiber, makes the water tank bracket meeting the using requirement to achieve the goal of lightweight. This paper studies the use of computer software for an automobile company in Haikou polypropylene - glass fiber material tank bracket for structural analysis, in order to verify the feasibility of using this new material tank car bracket.

\section{Establish Catia model car radiator bracket}

Establish the Catia model car radiator bracket mainly in two steps: determining the structural parameters; build a solid model tank bracket.

The model of the Car radiator support is divided into two steps: determining structure parameters; a water tank bracket model. The tank bracket of Haikou Automobile Company (polypropylene and glass fiber) is reinforced thin-walled structure and is complex, it adopts the integrated injection molding process. The total length of the water tank is $1138 \mathrm{~mm}$, height is $635 \mathrm{~mm}$, is composed of upper and lower two beams, two longitudinal beams and the side webs.

According to the simple principle and the drawings which the Haikou automotive trim Co. Ltd. provided, and then combined with the radiator support material, the solid modeling of automobile radiator bracket uses professional software CATIA. Car radiator support entity model is shown in figure 1.

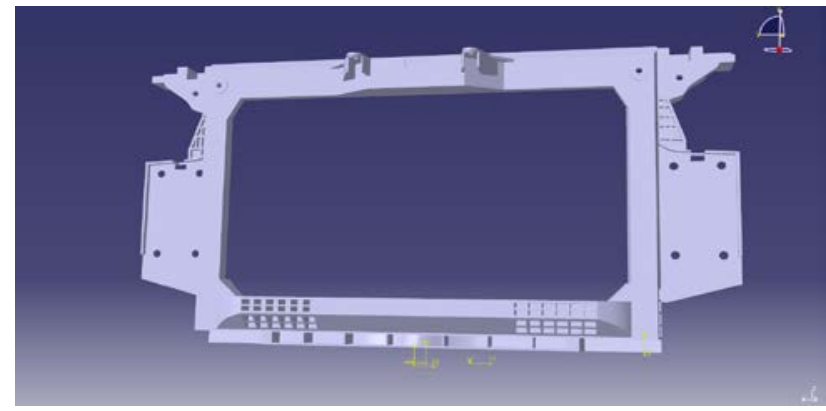

Figure 1: Car radiator support entity model of frontal.

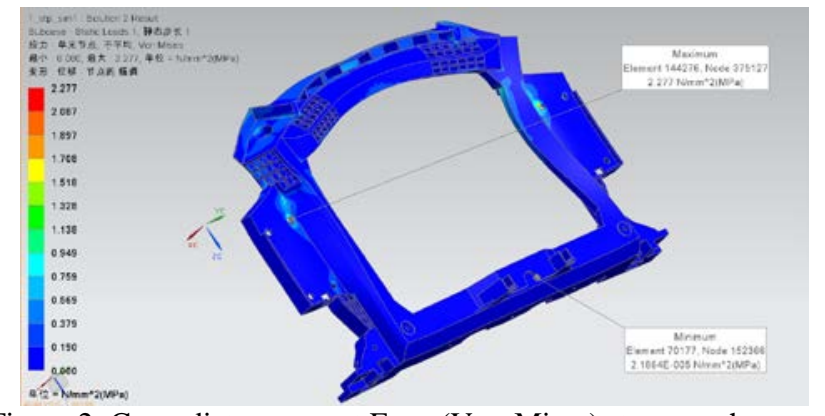

Figure 2: Car radiator support Feng (Von-Mises) stress nephogram 


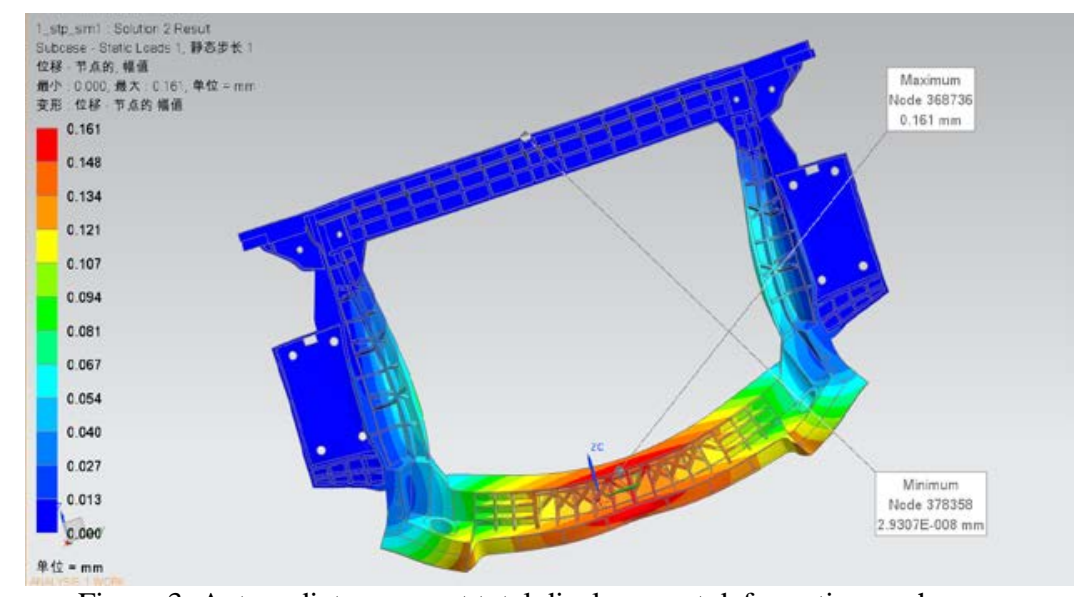

Figure 3: Auto radiator support total displacement deformation nephogram.

\section{Establish the finite element model of the automobile water tank bracket}

The water tank support entity model input UG software, will switch to the advanced UG simulation module, using the NX Nastran solver, to create the finite element model of the environment. The establishment of the finite element model is generally divided into the following steps: define material properties, defined cell properties etc. [1].

\section{The finite element static analysis of tank bracket}

After completing the grid division, constraint handling and load, chooses the appropriate solver to solve the finite element static analysis of the water tank bracket. Upon completion of auto radiator support is completed; acquire von stress distribution nephogram and displacement deformation nephogram.[2]. As shown in figure 2, figure 3.

From the point of von stress distribution, stress of the water tank bracket population distribution is uniform. Maximum stress appears in the left and right sides of near offset mounting holes in web, the obvious stress concentration, the maximum stress is 2.277 MPa. And Water tank bracket used in polypropylene, glass fiber material yield limit is $121 \mathrm{MPa}$.[3].Therefore, water tank bracket can satisfy the strength requirement.

The stiffness of the structure is mainly for its displacement deformation under the action of external loading. From the total displacement deformation nephogram of tank bracket, we can see that the maximum displacement occurred in the beam position on the middle, its displacement of the size is $0.161 \mathrm{~mm}$, almost no distortion. This illustrates the overall stiffness of the water tank bracket can be meet the design requirements.

\section{Finite element modal analysis of the water tank bracket}

Free vibration modal analysis is a linear analysis, a kind of approximate approach for the analysis of structural dynamic characteristics. In UG software, the modal analysis process is divided into four steps, namely, to establish the finite element model, constraint and load, extension, modal, and processing.[4].

Based on the earlier extended modal in the solution before, determine the required number of modal order solution.[5]. Extension mode, this paper calculated the first ten order modes, but only to take the first four order modal for reference. In the extracted solution automobile water tank bracket and the inherent frequency and vibration mode. Each order frequency, vibration mode results as shown in table1. 
Table 1. Table 1: Analysis and calculation results of modal car radiator support.

\begin{tabular}{|c|c|c|c|}
\hline \multirow{3}{*}{ Order } & Natural frequency & Vibration & The maximum \\
\hline & $/ \mathrm{Hz}$ & characteristics & deformation \\
\hline & & & $/ \mathrm{mm}$ \\
\hline $\mathrm{a}$ & 72 & The beam is bent in the direction of $\mathrm{X}$ & 0.825 \\
\hline $\mathrm{b}$ & 101 & The beam is bent in the direction of $\mathrm{Y}$ & 0.899 \\
\hline C & 127 & The beam is bent in the direction of $\mathrm{Z}$ & 1.215 \\
\hline \multirow[t]{2}{*}{ d } & 178 & Torsion beam under bending $+\mathrm{Z}$ direction & 0.768 \\
\hline & & Y direction & \\
\hline
\end{tabular}

Can be seen from table 1, car tank bracket of fourth-order modal frequencies corresponding mode shapes are local vibration, no water tank bracket the overall vibration, and fourth-order elastic modal frequency distribution in the range of $70 \mathrm{~Hz} \sim 280 \mathrm{~Hz}$.

According to the calculated results, combined with the natural frequencies and vibration modes of the water tank bracket can be seen, the first order natural frequency of car radiator support is greater than the excitation frequency range of vehicles, also higher than the natural frequency of the non sprung mass, is higher than the frequency of engine idle speed. As a result, the water tank bracket meets the requirement.

\section{Using ANSYS to analyze the water tank bracket}

To make our results more persuasive, we in the use of UG software for the tank after stent for structure analysis, ANSYS software is used to again structure analysis.

Through ANSYS software analysis, we obtain the overall stress diagram and the overall displacement diagram, as shown in figure 4, figure 5.

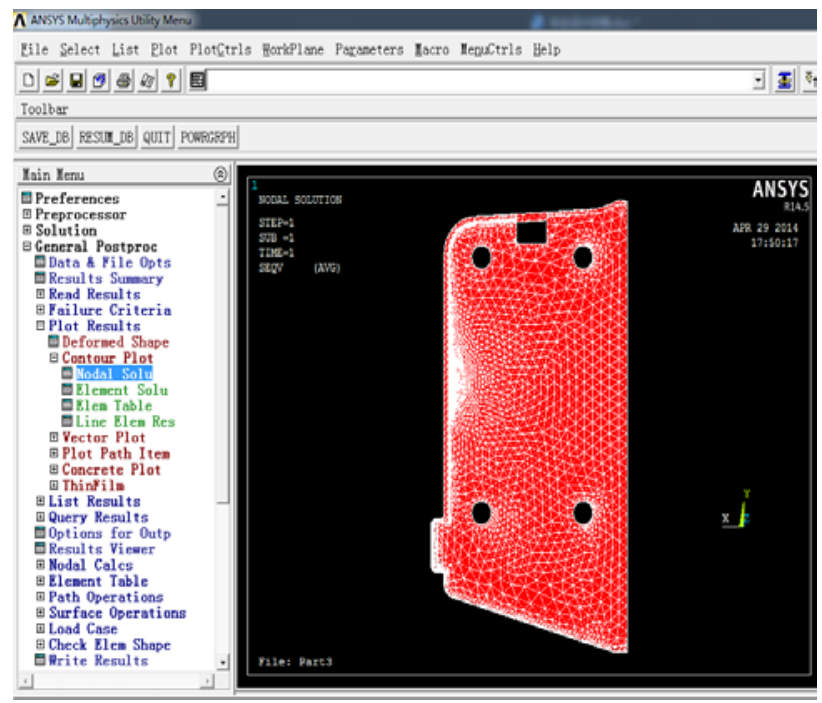

Figure 4: Stress diagram

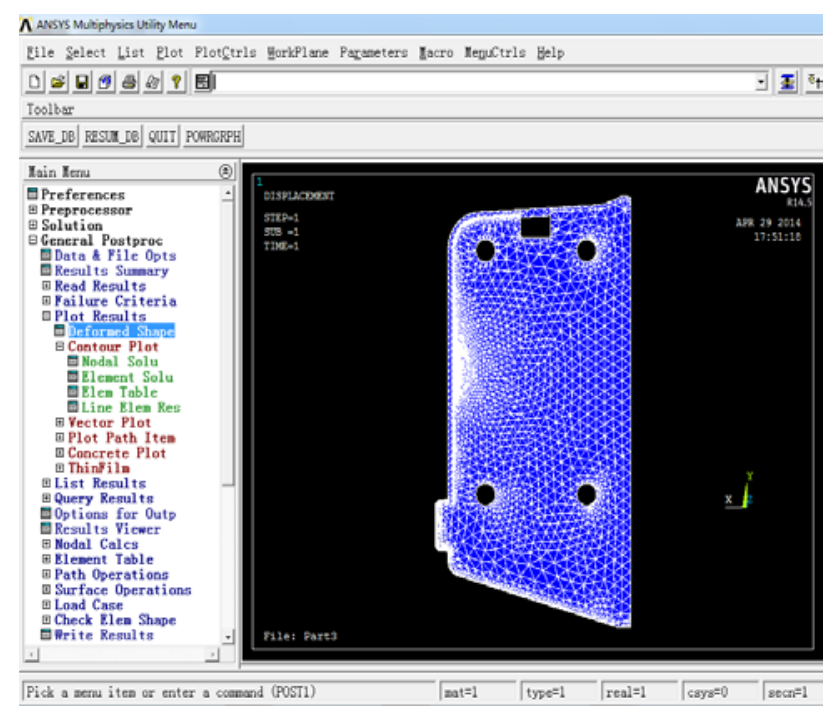

Figure 5: Displacement diagram.

Through the finite element analysis of ANSYS, two graphs obtained above, a stress, a displacement map. Because of the bolt hole is used as a fixed point, and plus force is slightly small, so is a pair of no displacement and stress deformation diagram. So, can also explain the tank bracket can satisfy the car use, the material strength and stiffness can be used as a source of water tank bracket manufacturing, meet the lightweight requirements, can improve the economic benefits. 


\section{Conclusions}

According to the computer using Catia, UG and ANSYS software to Haikou some automotive trim company production of new polymer materials tank bracket structure analysis results show that the new material of water tank support not only satisfy the use requirement, and compared with the traditional materials (such as steel, etc.) make stents can save raw materials, improve product performance and reduce the molding shrinkage rate, improve the heat distortion temperature, as well as the realization of auto lightweight, etc.

Application and development of the new material in the vehicle automobile radiator bracket is in accordance with the theme of security, environmental, and energy saving. On China's economy especially the research and application of technology has an important significance and broad application prospects of economic development in Hainan area, will greatly promote the development of vehicle design, produce significant economic and social benefits.

\section{Acknowledgement}

This research was financially supported by Production-teaching-research combination of Hainan province special funds (CXY20130048), Extensibility project of Hainan University (HD-KYH-2014079) and The important project of construction disciplines to the Midwest in Hainan university (ZXBJH-XK015). And Mei Li is the corresponding author in this article.

\section{References}

[1]. Wang, D.F. \& Huang, B., CATIA V5 Machine (Car) Product CAD/CAE/CAM Mastering Tutorial. China Communications Press: Beijing, 2007.3-7.

[2]. Tian, H.T., Disc brake vibration and noise analysis and research based on ANSYS. Wuhan University of Technology, 2008.

[3]. Juan, J., Based on UG modal analysis of engine bracket. Jiangxi Science, (01), pp. 66-69, 2011.

[4]. Bang, Q., Finite element analysis of the Formula SAE car frame structure strength optimization based on ANSYS. Henan University of Science \& Technology, 2013.

[5]. Wang, Q.C., Static and dynamic finite element analysis and structure improvement of the frame of CDW3100 dump truck. Chongqing University, 2008. 